\title{
NOTE ON AN INTEGRAL INEQUALITY FOR CONCAVE FUNCTIONS
}

\author{
HORST ALZER
}

Abstract. We prove: Let $p \in C^{2}[a, b]$ be non-negative and concave, and let $f \in$ $C^{2}[a, b]$ with $f(a)=f(b)=0$. Then

$$
\left(\int_{a}^{b} p(x)\left(f^{\prime}(x)\right)^{2} d x\right)^{2} \leq \int_{a}^{b} p(x)(f(x))^{2} d x \int_{a}^{b} p(x)\left(f^{\prime \prime}(x)\right)^{2} d x
$$

Moreover, we determine all cases of equality.

In a recently published article L. - C. Shen [3] presented some interesting integral inequalities involving polynomials. To prove his results Shen made use of an identity which we state here in a slightly modified form.

Lemma. Let $p \in C^{2}[a, b]$ and $f \in C^{2}[a, b]$. Then we have for all positive real numbers $\mathcal{L}$ :

$$
\begin{aligned}
& 2 \int_{a}^{b} p(x)\left(f^{\prime}(x)\right)^{2} d x=\int_{a}^{b}\left[\mathcal{L} p(x)+p^{\prime \prime}(x)\right](f(x))^{2} d x \\
+ & \frac{1}{\mathcal{L}} \int_{a}^{b} p(x)\left(f^{\prime \prime}(x)\right)^{2} d x-\frac{1}{\mathcal{L}} \int_{a}^{b} p(x)\left[\mathcal{L} f(x)+f^{\prime \prime}(x)\right]^{2} d x+C,
\end{aligned}
$$

where

$$
C=2 p(b) f(b) f^{\prime}(b)-2 p(a) f(a) f^{\prime}(a)+p^{\prime}(a)(f(a))^{2}-p^{\prime}(b)(f(b))^{2} .
$$

It is the aim of this note to show how the Lemma can be applied to establish a new integral inequality for concave functions which is related to the classical inequality

$$
\left(\int_{0}^{\infty}\left(f^{\prime}(x)\right)^{2} d x\right)^{2} \leq 4 \int_{0}^{\infty}(f(x))^{2} d x \int_{0}^{\infty}\left(f^{\prime \prime}(x)\right)^{2} d x
$$

Received February 13, 1995.

1991 Mathematics Subject Classification. Primary 26D10, secondary 26D15.

Key words and phrases. Integral inequality involving a function and its derivatives, concave functions. 
due to G.H. Hardy, J.E. Littlewood and G. Pólya [2, p.187].

Theorem. Let $p \in C^{2}[a, b]$ be non-negative and concave, and let $f \in C^{2}[a, b]$ with $f(a)=f(b)=0$. Then

$$
\left(\int_{a}^{b} p(x)\left(f^{\prime}(x)\right)^{2} d x\right)^{2} \leq \int_{a}^{b} p(x)(f(x))^{2} d x \int_{a}^{b} p(x)\left(f^{\prime \prime}(x)\right)^{2} d x .
$$

If $p \not \equiv 0$ and $f \not \equiv 0$, then the sign of equality holds in (2) if and only if $p$ is linear and $f(x)=A \cos \frac{k \pi x}{b-a}+B \sin \frac{k \pi x}{b-a}$, where $A, B \in \mathbb{R},(A, B) \neq(0,0)$, and $k \in \mathbb{N}$ such that $A \cos \frac{k \pi a}{b-a}+B \sin \frac{k \pi a}{b-a}=0$.

Proof. Let $p \not \equiv 0$ and $f \not \equiv 0$. Since $f(a)=f(b)=0$ we conclude that $f^{\prime \prime} \not \equiv 0$, which implies

$$
\mathcal{L}_{0}=\left(\int_{a}^{b} p(x)\left(f^{\prime \prime}(x)\right)^{2} d x / \int_{a}^{b} p(x)(f(x))^{2} d x\right)^{1 / 2}>0 .
$$

If we replace in $(1) \mathcal{L}$ by $\mathcal{L}_{0}$, then we get

$$
\begin{aligned}
& 2 \int_{a}^{b} p(x)\left(f^{\prime}(x)\right)^{2} d x \\
= & 2\left(\int_{a}^{b} p(x)(f(x))^{2} d x \int_{a}^{b} p(x)\left(f^{\prime \prime}(x)\right)^{2} d x\right)^{1 / 2} \\
+ & \int_{a}^{b} p^{\prime \prime}(x)(f(x))^{2} d x-\frac{1}{\mathcal{L}_{0}} \int_{a}^{b} p(x)\left[\mathcal{L}_{0} f(x)+f^{\prime \prime}(x)\right]^{2} d x .
\end{aligned}
$$

Since $p$ is concave we have $p^{\prime \prime} \leq 0$, so that (3) leads to inequality (2).

We discuss the cases of equality. A simple calculation reveals that the sign of equality holds in (2) if $p(x)=c_{0}+c_{1} x$ and $f(x)=A \cos \frac{k \pi x}{b-a}+B \sin \frac{k \pi x}{b-a}$, where $A, B \in \mathbb{R}, k \in \mathbb{N}$ satisfy $\mathrm{A} \cos \frac{k \pi a}{b-a}=-B \sin \frac{k \pi a}{b-a}$.

If equality is valid in (2), then we obtain from (3) that

$$
\int_{a}^{b} p(x)\left[\mathcal{L}_{0} f(x)+f^{\prime \prime}(x)\right]^{2} d x=0
$$

and

$$
\int_{a}^{b} p^{\prime \prime}(x)(f(x))^{2} d x=0
$$

To determine $f$ we conclude from (4) that

$$
\mathcal{L}_{0} f(x)+f^{\prime \prime}(x)=0
$$

which leads to

$$
f(x)=A \cos \left(\sqrt{\mathcal{L}_{0}} x\right)+B \sin \left(\sqrt{\mathcal{L}_{0}} x\right) ; \quad A, B \in \mathbb{R} .
$$


From $f(a)=f(b)=0$ we get

$$
\begin{aligned}
& A \cos \left(\sqrt{\mathcal{L}_{0}} a\right)+B \sin \left(\sqrt{\mathcal{L}_{0}} a\right)=0 \\
& A \cos \left(\sqrt{\mathcal{L}_{0}} b\right)+B \sin \left(\sqrt{\mathcal{L}_{0}} b\right)=0
\end{aligned}
$$

Since $(A, B) \neq(0,0)$ we conclude from $(6)$ that

$$
\sin \left(\sqrt{\mathcal{L}_{0}}(b-a)\right)=0
$$

which implies

$$
\sqrt{\mathcal{L}_{0}}=k \pi /(b-a), \quad k \in \mathbb{N}
$$

Thus, we have

$$
f(x)=A \cos \frac{k \pi x}{b-a}+B \sin \frac{k \pi x}{b-a} ; \quad A, B \in \mathbb{R}, \quad(A, B) \neq(0,0),
$$

with

$$
A \cos \frac{k \pi a}{b-a}+B \sin \frac{k \pi a}{b-a}=0
$$

And, from (5) and (7) we obtain $p^{\prime \prime} \equiv 0$, which implies that $p$ is linear.

Remarks. 1) Inequality (2) is in general not true if the assumption " $p$ is concave" will be dropped. For instance, if we set $a=0, b=\pi, p(x)=x^{2}$ and $f(x)=\sin (x)$, then (2) holds with" $>$ " instead of " $\leq$ ".

2) Concerning further integral inequalities for concave functions we refer to [1] and the references therein.

\section{References}

[1] J. L. Brenner and H. Alzer, "Integral inequalities for concave functions with applications to special functions," Proc. Roy. Soc. Edinburgh Sect.A, 118(1991), 173-192.

[2] G. H. Hardy, J. E. Littlewood and G. Pólya, Inequalities, Cambridge Univ. Press, 1934.

[3] L. -C. Shen, "Comments on an $L^{2}$ inequality of A. K. Varma involving the first derivative of polynomials," Proc. Amer. Math. Soc. 111(1991), 955-959.

Morsbacher Str. 10, 51545 Waldbröl, Germany. 